\title{
Dimensions of the Great Intrathoracic Arteries of Early Mouse Fetuses of the C57BL/6 Strain
}

\author{
Stefan H. Geyer, Barbara Maurer, Kathrin Dirnbacher and Wolfgang J. Weninger*
}

Medical University of Vienna, Center for Anatomy and Cell Biology, Waehringer Str. 13, A-1090 Vienna, Austria

\begin{abstract}
Careful analysis of the morphological phenotype of malformed mouse fetuses is an essential step in researching the genesis of cardiovascular malformations. The detection of arterial stenosis and dilation requires metric analysis and reference data defining the normal dimensions of the fetal arteries. Since the genetic background of inbred strains strongly influences the dimensions of the arteries, reference data are strain specific. We aimed at creating objective reference data for diagnosing the stenosis and dilation of important segments of the great intrathoracic arteries of early mouse fetuses bred on the C57BL/6 background. Employing the high-resolution episcopic microscopy (HREM) technique, we created volume data and three-dimensional (3D) computer models of the great intrathoracic arteries of 30 mouse fetuses of developmental stage 22 according to Theiler (TS22). We then defined reproducible measurement positions along the ascending aorta, the pulmonary trunk, the ductus arteriosus and the descending aorta and used a recently published protocol to measure the diameter of the lumen of the arteries at these positions. We present the values obtained for the diameters as measured in $\mu \mathrm{m}$. We also present the relation of the diameter measured for each blood vessel and the diameters of the lumen of the ascending aorta and pulmonary trunk. The measurements and relative values we provide objectively define the dimensions of important segments of the great intrathoracic arteries of normally developed TS 22 mouse fetuses of the $\mathrm{C} 57 \mathrm{BL} / 6$ strain. They will function as reliable reference data for diagnosing stenosis and dilation of the measured segments in malformed fetuses.
\end{abstract}

Keywords: Aortic arch arteries, biometry, development, embryo, episcopic imaging, morphometry, pharyngeal arch arteries, 3D imaging.

\section{INTRODUCTION}

$1 \%$ of all newborns suffer from malformations of the cardiovascular system $[1,2]$. For researching the genetic factors underlying these malformations model organisms are employed. The most important model organism is the mouse. Careful analysis of the morphological phenotype of transgenic mice lacking functional regulatory developmental factors is essential for revealing gene function. However, gene deletion often causes prenatal death of affected individuals. In such cases the morphological phenotype of embryos or fetuses must be analysed.

In transgenic or manipulated mouse embryos and fetuses, structural cardiac defects and missing, additional, or aberrantly running blood vessels can be easily diagnosed by employing various modern three-dimensional (3D) reconstruction techniques [3-11]. The diagnosis of blood vessel stenosis or dilation (i.e. aortic isthmus stenosis, etc.) is more demanding. On the one hand it requires a technique, which permits measuring blood vessel diameters exactly perpendicular to the longitudinal axis of the blood vessel. On the other hand it requires reference data, which objectively define the dimensions of the blood vessels of wild type mouse embryos and fetuses.

A recently published protocol permits measuring the diameter of the great intrathoracic arteries of embryos of

\footnotetext{
*Address correspondence to this author at the Medical University of Vienna, Center for Anatomy and Cell Biology, Waehringer, str. 13, A-1090 Vienna, Austria; Tel: +43-1-4277-61136; Fax: +43-1-4217-61118;

E-mail:wolfgang.weninger@meduniwien.ac.at
}

model organisms in a reliable and objective way $[12,13]$. Using this protocol, first reference data for diagnosing the stenosis of the great intrathoracic arteries of early mouse fetuses have been generated and published. These data show that the dimensions of the great intrathoracic arteries of different mouse strains differ considerably. Thus the blood vessel dimensions of mutants bred on a distinct genetic background must be compared with the blood vessel dimensions of wild types having the same genetic background.

One of the most popular mouse strains for researching cardiovascular development is the C57BL/6-strain. Up to now no reference data defining the dimensions of the great intrathoracic arteries of early fetuses of developmental stage 22 according to Theiler [14] are available for this strain.

This study aims at presenting reference data, which objectively define the lumen diameters of the most important segments of the great intrathoracic arteries of mouse fetuses of developmental stage 22 according to Theiler, which were bred on the C57BL/6 background.

\section{MATERIAL AND METHODOLOGY}

We analysed 30 mouse (Mus musculus) embryos of the C57BL/6 strain (Biomodels Austria). The embryos were harvested on day 14.5 post conceptionem $(\mathrm{dpc})$ and staged according to Theiler [14]. Only embryos showing the external features characteristic for developmental stage 22 (TS22) were analysed. These features were: separated, individual fingers in the forefoot plate, deep indentations 
between the developing toes in the hindfoot, and covering of half of the auditory meatus by the pinna.

The embryos were prepared for episcopic volume data generation with the High-Resolution Episcopic Microscopy (HREM) technique [9]. We followed a slightly modified standard protocol for generating 3D models of the late embryonic and early fetal heart and great intrathoracic arteries [13]. Immediately after harvesting and staging, we dissected the embryos into 3 parts - head, thorax, and abdomen. The thoraces were fixed in $4 \%$ paraformaldehyde buffered with PBS. After $48 \mathrm{~h}$ they were transferred into $70 \%$ ethanol, in which they were stored for several weeks.

For generating digital volume data, we dehydrated the specimens in ethanol and embedded them in resin. In brief, from the $70 \%$ ethanol, we transferred the specimens into $80 \%$ ethanol (for $8 \mathrm{~h}$ ), then into $90 \%$ (over night) and finally into $96 \%$ (for $8 \mathrm{~h}$ ). Then they were transferred into an infiltration solution, which was composed of solution A of a resin embedding kit (JB4, Polysciences Inc., Germany), and benzoyl peroxide, plasticized $(1.25 \mathrm{~g} / 100 \mathrm{ml})$, dyed with eosin (eosin-spritlöslich $0.4 \mathrm{~g} / 100 \mathrm{ml}$, Waldeck GmbH, Germany). The specimens were infiltrated for 24 hours. The solution was changed twice. Finally the specimens were embedded in resin dyed with eosin $(0.4 \mathrm{~g} / 100 \mathrm{ml} \mathrm{JB} 4)$.

The resin blocks were allowed to harden for at least 4 days. Then, they were mounted on a HREM apparatus and subjected to HREM volume data generation [8, 9, 15]. HREM data generation resulted in digital volume data with voxel sizes of $1.07 \mu \mathrm{m} \times 1.07 \mu \mathrm{m} \times 2 \mu \mathrm{m}$. In these data we manually traced the lumina of the great intrathoracic arteries thereby creating binary data. From the binary data we created surface 3D models.

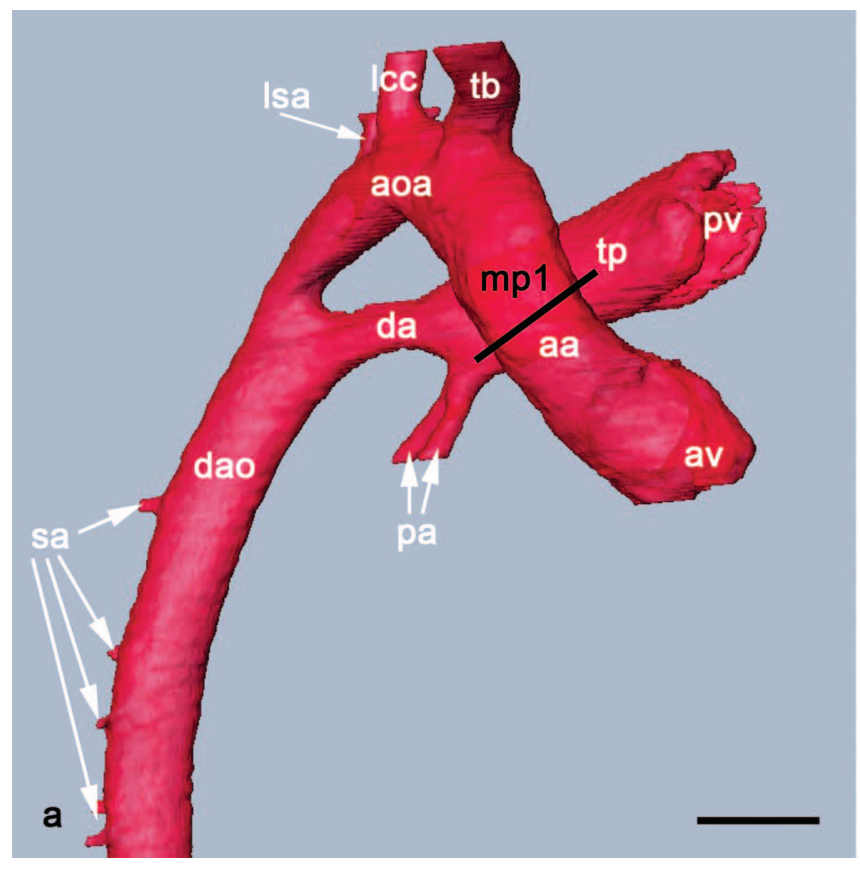

After screening the topology of the great intrathoracic arteries we used the 3D models for measuring the diameters of important arteries. For performing the metric analysis we followed a protocol recently developed in our laboratory [13].

With the aid of the 3D models we defined comparable positions along the blood vessel trees. The positions were: mp1: Ascending aorta halfway between the aortic valve and the origin of the brachiocephalic trunk; mp2: descending aorta distal to the connection with the ductus arteriosus; $\mathrm{mp} 3$ : descending aorta proximal to the origin of the first pair of segmental arteries; mp4: ductus arteriosus halfway between its connection with the descending aorta and the origin of the pulmonary arteries. mp5: pulmonary trunk halfway between the opening of the pulmonary valve and the origin of the pulmonary arteries (Fig. 1).

At each of these positions we defined a virtual plane, which cut through the original volume data perpendicularly to the longitudinal axis of the artery (Fig. 2). In this plane, we displayed a scale bar and captured a screen shot of the virtual section. The screenshot was loaded in the Image J software (National Institute of Health) and the perimeter of the lumen was traced and measured. The lumen diameter was calculated from the perimeter. Then, we calculated the ratios of each measured blood vessel diameter and the diameters of the ascending aorta and the pulmonary trunk.

Binarisation, 3D modelling, 3D visualisation, topological, and metric analysis were performed using the Amira 5.0 software (Mercury Computer Systems, Germany), running on a PC featured with Intel Xeon X5355 Processor $(2,66 \mathrm{GHz}), 32 \mathrm{~GB}$ RAM, and ATI FireGL V7350 (1GB) graphic board. For statistics, we utilized the MS Excel (Microsoft) and SPSS (SPSS Inc.) software packages.

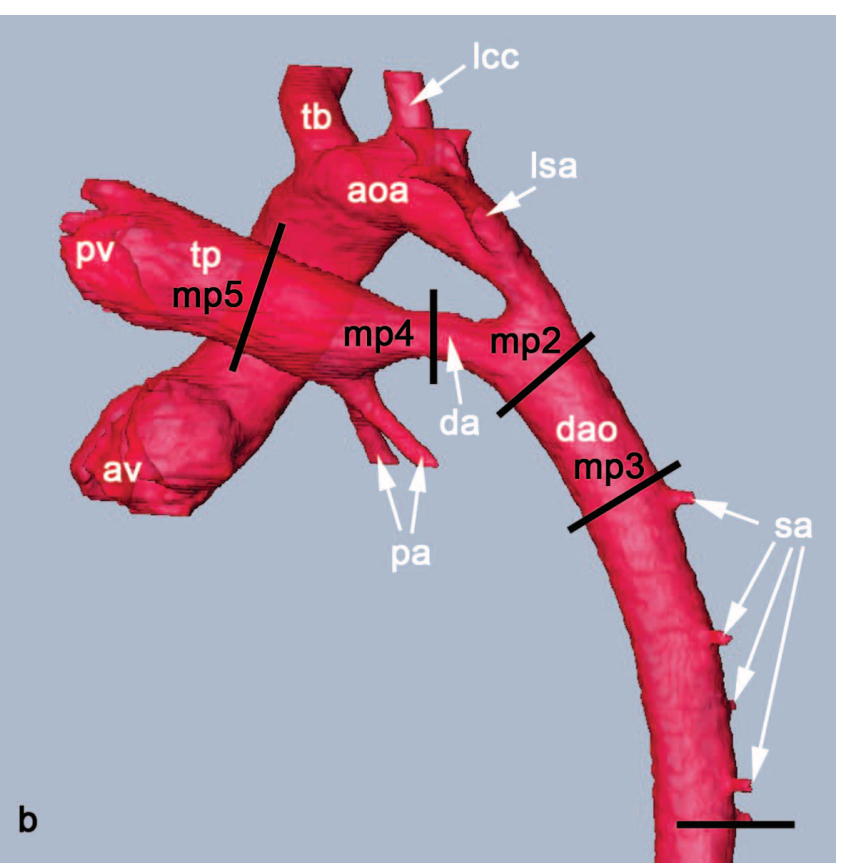

Fig. (1). Measuring positions along the great intrathoracic arteries of a C57BL/6 mouse fetus of TS 22. a. 3D surface model of the great intrathoracic arteries from right. b. 3D surface model of the great intrathoracic arteries from left. aa $=$ ascending aorta; aoa $=$ aortic arch; av $=$ aortic valve; $\mathrm{da}=$ ductus arteriosus; dao = descending aorta; $1 \mathrm{cc}=$ left common carotid artery; lsa $=$ left subclavian artery; pa $=$ pulmonary arteries; $\mathrm{pv}=$ pulmonary valve; $\mathrm{sa}=$ segmental arteries; $\mathrm{tb}=$ brachiocephalic trunk; $\mathrm{tp}=$ pulmonary trunk. $\mathrm{mp} 1=$ measuring position $1 ; \mathrm{mp} 2$ $=$ measuring position $2 ; \mathrm{mp} 3=$ measuring position $3 ; \mathrm{mp} 4=$ measuring position $4 ; \mathrm{mp} 5=$ measuring position 5. Scalebars $=200 \mu \mathrm{m}$. 


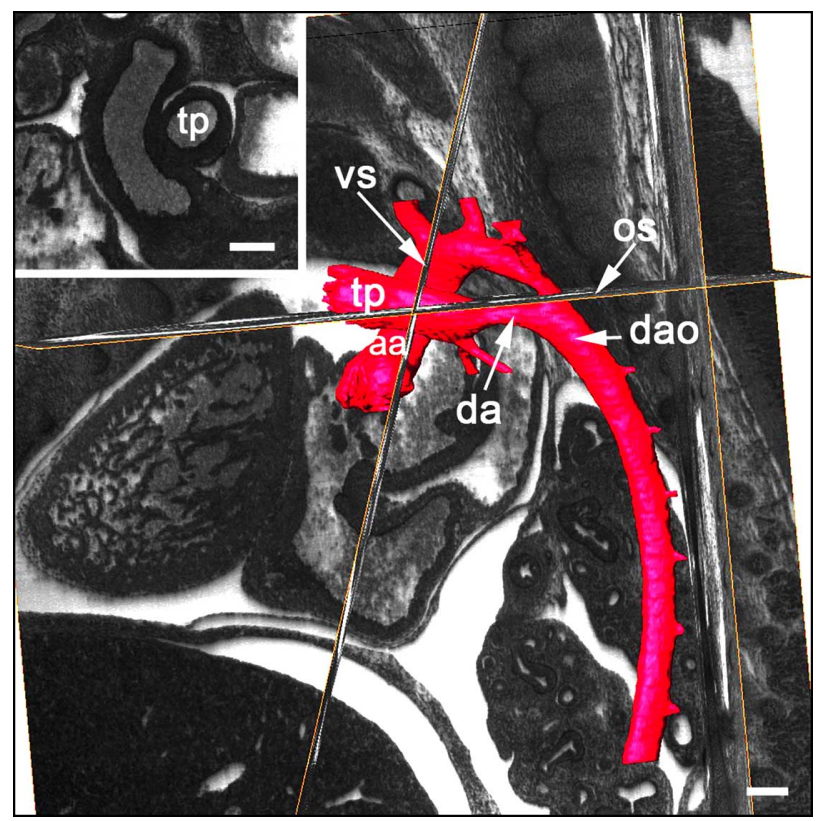

Fig. (2). Definition of the correct plane for measuring the diameter of the pulmonary trunk (tp). Note the orientation of the original sections (os). The inlay shows the virtual section plane (vs), which was defined to cut through the volume data perpendicularly to the longitudinal axis of the pulmonary trunk. aa = ascending aorta; $\mathrm{da}=$ ductus arteriosus; dao $=$ descending aorta. Scalebars $=200 \mu \mathrm{m}$.

\section{RESULTS}

\section{Measurements in $\mu \mathrm{m}$}

The mean diameter of the ascending aorta halfway between the aortic valve and the origin of the brachiocephalic trunk ( $\mathrm{mp}$ 1) was $157.85 \mu \mathrm{m}(116.65 \mu \mathrm{m}-252.38 \mu \mathrm{m})$. The mean diameter of the descending aorta distal to the connection with the ductus arteriosus (mp 2) was $169.81 \mu \mathrm{m}(127.14 \mu \mathrm{m}-$ $214.50 \mu \mathrm{m})$. The mean diameter of the descending aorta proximal to the origin of the first pair of segmental arteries $(\mathrm{mp}$ 3) was $177.75 \mu \mathrm{m}(108.45 \mu \mathrm{m}-248,61 \mu \mathrm{m})$. The mean diameter of the ductus arteriosus halfway between the origin of the pulmonary arteries and its connection with the descending aorta (mp 4) was $118.14 \mu \mathrm{m}(50.33 \mu \mathrm{m}-158.54 \mu \mathrm{m})$. The mean diameter of the pulmonary trunk halfway between the opening of the pulmonary valve and the origin of the pulmonary arteries (mp 5) was $161.87 \mu \mathrm{m}(130.34 \mu \mathrm{m}-200.01 \mu \mathrm{m})($ Table 1, Fig. 3).

\section{Ratios}

The mean diameter of the ascending aorta halfway between the aortic valve and the origin of the brachiocephalic trunk (mp 1) measured 97.96\% (71.32\% $141.12 \%$ ) of the mean diameter of the pulmonary trunk. The mean diameter of the descending aorta distal to the connection with the ductus arteriosus (mp 2) measured $105.77 \%(75.17 \%-129.71 \%)$ of the mean diameter of the pulmonary trunk and $110.11 \%(70.70 \%-168.46 \%)$ of the mean diameter of the ascending aorta. The mean diameter of the descending aorta proximal to origin of the first pair of segmental arteries (mp 3) measured 111.24\% (64.50\% $154.97 \%$ ) of the mean diameter of the pulmonary trunk and $115.10 \%(68.54 \%-164.11 \%)$ of the mean diameter of the ascending aorta. The mean diameter of the ductus arteriosus halfway between the origin of the pulmonary arteries and its connection with the descending aorta (mp 4) measured $74.05 \%(30.22 \%-109.89 \%)$ of the mean diameter of the pulmonary trunk and $76.94 \%(32.85 \%-117.62 \%)$ of the mean diameter of the ascending aorta. The mean diameter of the pulmonary trunk halfway between the opening of the pulmonary valve and the origin of the pulmonary arteries (mp 5) measured $104.20 \%(70.86 \%-140.21 \%)$ of the mean diameter of the ascending aorta (Table 1, Fig. 4).

\section{DISCUSSION AND CONCLUSION}

For the first time, this study objectively defines the dimensions of the diameters of the lumina of the great intrathoracic arteries of C57BL/6-mouse embryos of developmental stage 22 according to Theiler. Such information is important for diagnosing stenosis and dilation of the great intrathoracic arteries in malformed fetuses as produced in gene deletion experiments and mutagenesis screens.

Our data are highly reliable, because we used a newly proposed and evaluated protocol for measuring diameters of blood vessels of embryos and fetuses of model organisms $[12,13]$. The protocol works with digital volume data and 3D computer models and permits the precise and reproducible identification of comparable measurement positions along the great intrathoracic arteries. It further fits for defining a virtual resection plane, which cuts through the digital volume data perpendicularly to the longitudinal axis of the blood vessel segment to measure. This is essential for performing correct measurements of blood vessel diameters. Finally it includes measuring the perimeter of the lumen of

Table 1. Mean Lumen Diameters of the Great Arteries of C57BL/6J Mouse Embryos of Developmental Stage 22 According to Theiler

\begin{tabular}{|c|c|c|c|c|c|c|}
\hline $\begin{array}{c}\text { Measuring } \\
\text { Positions (n=30) }\end{array}$ & $\begin{array}{c}\text { Lumen Diameter } \\
\text { in } \boldsymbol{\mu m} \text { (SD) }\end{array}$ & $\begin{array}{c}\text { Ratio with mp1 } \\
\text { (\%) (SD) }\end{array}$ & $\begin{array}{c}\text { Ratio with mp2 } \\
\text { (\%) (SD) }\end{array}$ & $\begin{array}{c}\text { Ratio with mp3 } \\
\text { (\%) (SD) }\end{array}$ & $\begin{array}{c}\text { Ratio with mp4 } \\
\text { (\%) (SD) }\end{array}$ & $\begin{array}{c}\text { Ratio with mp5 } \\
\text { (\%) (SD) }\end{array}$ \\
\hline \hline $\mathrm{mp} 1$ & $157.85(24.91)$ & 100 & $95(20)$ & $92(24)$ & $144(52)$ & $98(14)$ \\
\hline $\mathrm{mp} 2$ & $169.81(23.08)$ & $110(23)$ & 100 & $97(12)$ & $150(33)$ & $106(16)$ \\
\hline $\mathrm{mp} 3$ & $177.75(33.30)$ & $115(27)$ & $105(14)$ & 100 & $154(26)$ & $111(25)$ \\
\hline $\mathrm{mp} 4$ & $118.14(27.17)$ & $77(23)$ & $69(13)$ & $66(10)$ & 100 & $74(20)$ \\
\hline $\mathrm{mp} 5$ & $161.87(16.76)$ & $104(15)$ & $97(17)$ & $95(25)$ & $148(54)$ & 100 \\
\hline
\end{tabular}

$\mathrm{mp} 1=$ measuring position along the ascending aorta; $\mathrm{mp} 2=$ measuring position along the proximal part of the descending aorta $\mathrm{I}$; $\mathrm{mp} 3=\mathrm{measuring}$ position along the distal part of the descending aorta II; mp4 = measuring position along the ductus arteriosus; $\mathrm{mp} 5=$ measuring position along the pulmonary trunk, $\mathrm{SD}=$ standard deviation. 

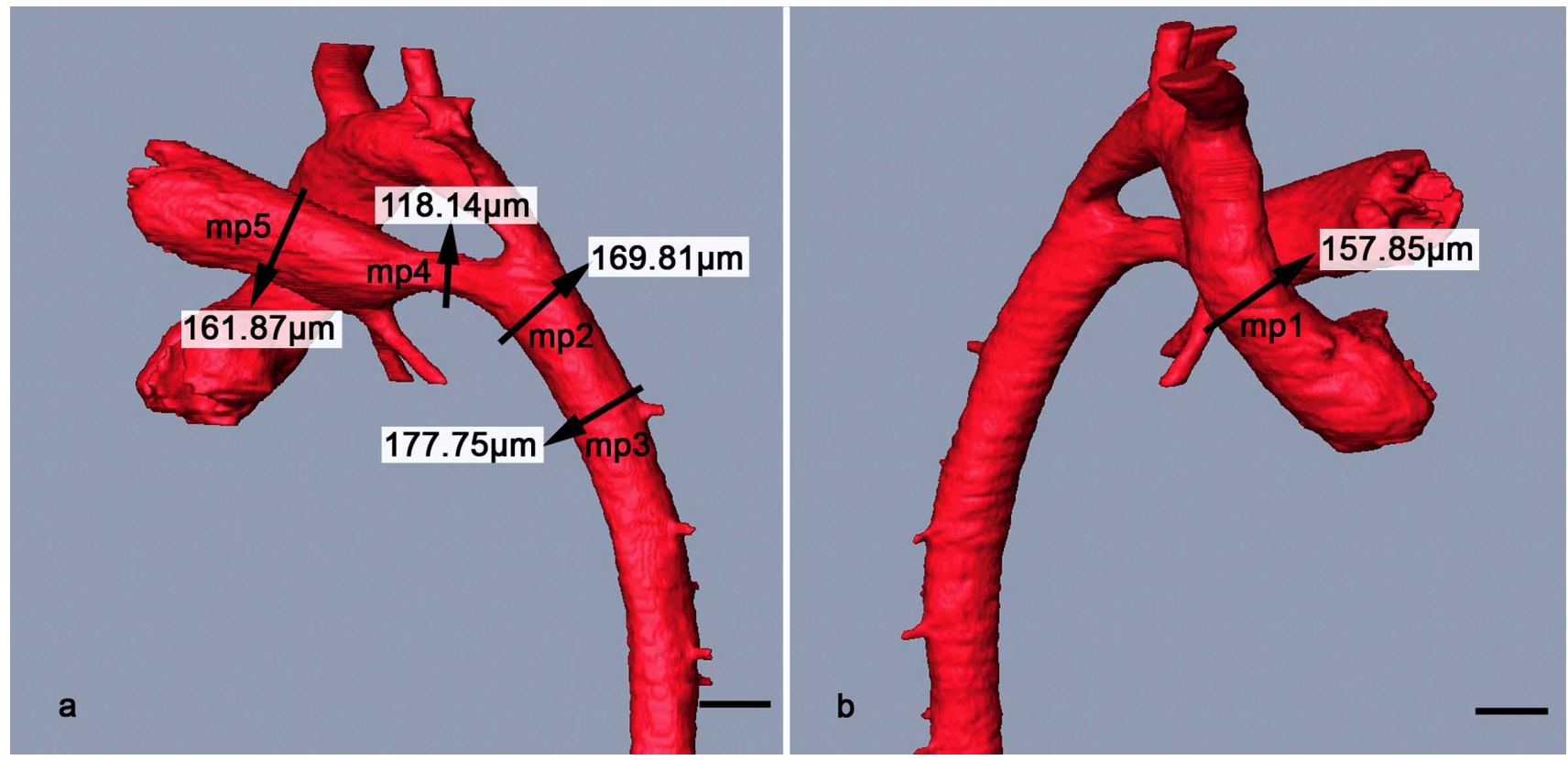

Fig. (3). Mean diameter of the lumen at positions mp1-mp5 (as measured in $\mu \mathrm{m}$ ). a. 3D surface model of the great intrathoracic arteries from right. b. 3D surface model of the great intrathoracic arteries from left. $\mathrm{mp} 1=$ measuring position 1 ; mp $2=$ measuring position 2 ; mp3 = measuring position $3 ; \mathrm{mp} 4=$ measuring position $4 ; \mathrm{mp} 5=$ measuring position 5 . Scalebars $=200 \mu \mathrm{m}$.

the blood vessels and calculating the diameter from the perimeter. This minimises the influence of inhomogeneous shrinkages of the various tissues of specimens during their fixation and irregularities of the lumen borders caused by these shrinkages.

For gathering the digital volume data, we employed the high resolution episcopic microscopy (HREM) technique [9]. HREM provides high quality 3D volume data with voxel sizes of $1.07 \times 1.07 \times 2 \mu \mathrm{m}^{3}$. Such data were shown to facilitate measurements of blood vessel diameters with a maximum error of $\pm 7 \mu \mathrm{m}$. Measuring the diameter of the lumen of the great intrathoracic arteries of early mouse fetuses this accounts for a maximal uncertainty of the reliability of the measurements of $\pm 5 \%$ [13].

Two factors have a strong negative influence on the interindividual comparability of measurements of lumen diameters with the protocol we used: 1) The lumen of blood vessels must be individually defined by the researcher
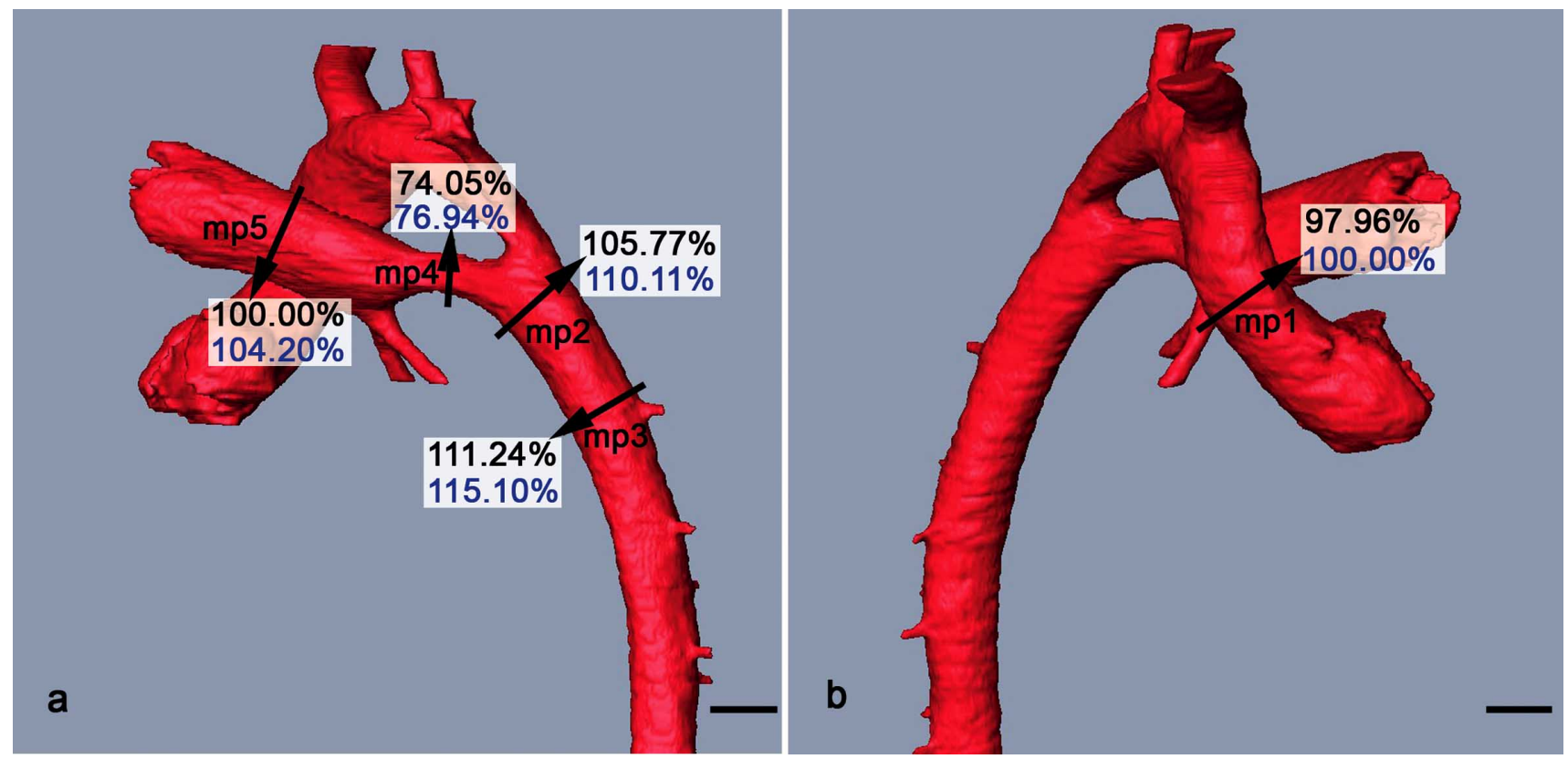

Fig. (4). Mean diameter of the lumen at positions mp1-mp5 in relation to mp5 (top number) and mp1 (bottom number). a. 3D surface model of the great intrathoracic arteries from right. b. 3D surface model of the great intrathoracic arteries from left. mp $1=\mathrm{measuring}$ position 1 ; $\mathrm{mp} 2=$ measuring position $2 ; \mathrm{mp} 3=$ measuring position $3 ; \mathrm{mp} 4=$ measuring position $4 ; \mathrm{mp} 5=$ measuring position 5 . Scalebars $=200 \mu \mathrm{m}$. 
performing the measurements. Due to subjective interpretation of blood vessel borders by different researchers the diameters measured for a blood vessel segment may show a difference of up to $28 \mu \mathrm{m}$ in data sets with voxel sizes of $1.07 \times 1.07 \times 1.07 \mu \mathrm{m}^{3}$ [12] 2) The embedding of the specimen into resin causes shrinkages of the tissues of the specimen. Due to different specimen processing protocols, the amount of these shrinkages may vary considerably.

The negative influence of these factors can be minimised by using ,relative values“ instead of ,absolute values“ [12]. We therefore do not only simply provide measurements of the diameters of the lumen of the arteries. We also calculated the relation between each measured diameter, and the diameter of the lumen of the ascending aorta and pulmonary trunk and provide the obtained values as relative values. In addition, we provide the ratios of all measured arteries in the table.

Another positive effect of the use of relative values is that they ensure that our data are comparable to measurements obtained by using all modern imaging techniques designed to produce digital volume data. This includes optical projection tomography (OPT) [16, 17], micro-Magnetic resonance imaging $(\mu \mathrm{MRI})[18,19]$, and micro-computed tomography $(\mu \mathrm{CT})[6,20,21]$.

Large numbers of mutated mouse strains are generated in projects, such as the European Conditional Mouse Mutagenesis Program (http://www.knockoutmouse.org/about/euc omm), the North American Conditional Mouse Mutagenesis Project (http://www.norcomm.org/index.htm), or the Knock Out Mouse Project (http://www.komp.org/). The majority of the mutants are created on a C57BL/6 background. Therefore for identifying pathologic phenotypes created in the scope of these projects, reference data defining the normal morphology of C57BL/6 mice are urgently required.

In many mutated strains homozygous individuals die before birth. In these cases embryos must be phenotyped. The best time for doing this is around $14.5 \mathrm{dpc}$ (approximately TS22). The reason is, that TS22 is the edge of the embryonic to the fetal period. In TS22 embryos the so called "fetal circulation" is established, wherefore malformations of the great intrathoracic arteries can be diagnosed correctly.

Reference data defining the dimensions of the great intrathoracic arteries do already exist for 14.5 and $15.5 \mathrm{dpc}$ mouse embryos bred on PARKES and OF1 backgrounds [13]. But since recent findings indicate, that the dimensions of the great intrathoracic arteries can differ between mice bred on various genetic backgrounds objective definitions of the dimensions of the great intrathoracic arteries of TS22 embryos bred on the C57BL/6 background are required. This study presents such data.

At present, measurements of the ascending and descending aorta and the pulmonary trunk do exist for both, TS22 OF1 and C57BL/6 mice. Direct comparisons of the data reveal significant differences of the diameters (as measured in $\mu \mathrm{m}=$ absolute values) of the ascending aorta $(\mathrm{p}=0.017)$ and the pulmonary trunk $(\mathrm{p}=0.017)$. In contrast, comparisons of the ratios of the measured arteries (descending aorta/ascending aorta, ascending aorta/ pulmonary trunk, descending aorta/pulmonary trunk) do not reveal significant differences between the two strains. We think the reason for this lies in the different interpretations of the borders of the blood vessel lumina by the two researchers, who performed the measurements. This bias is eliminated by comparing ratios. Thus from the comparisons of the relative values we conclude that the hemodynamic conditions in the aorta and pulmonary trunk of TS22 embryos of the OF1 and C56B1/6 strains are largely similar.

\section{ACKNOWLEDGEMENT}

Declared none.

\section{CONFLICT OF INTEREST}

Declared none.

\section{REFERENCES}

[1] Pierpont ME, Basson CT, Benson DW, et al. Genetic basis for congenital heart defects: current knowledge: a scientific statement from the American Heart Association Congenital Cardiac Defects Committee, Council on Cardiovascular Disease in the Young: endorsed by the American Academy of Pediatrics. Circulation 2007; 115: 3015-38.

[2] Hoffman JI, Kaplan S. The incidence of congenital heart disease. J Am Coll Cardiol 2002; 39: 1890-900.

[3] Männer J, Thrane L, Norozi K, Yelbuz TM. High-resolution in vivo imaging of the cross-sectional deformations of contracting embryonic heart loops using optical coherence tomography. Dev Dyn 2008; 237: 953-61.

[4] Schneider JE, Bhattacharya S. Making the mouse embryo transparent: identifying developmental malformations using magnetic resonance imaging. Birth Defects Res C Embryo Today 2004; 72: 241-9.

[5] Pieles G, Geyer SH, Szumska D, et al. microMRI-HREM pipeline for high-throughput, high-resolution phenotyping of murine embryos. J Anat 2007; 211: 132-7.

[6] Metscher BD. MicroCT for developmental biology: A versatile tool for high-contrast 3D imaging at histological resolutions. Dev Dyn 2009; 238: 632-40.

[7] Geyer SH, Mohun TJ, Weninger WJ. Visualizing vertebrate embryos with episcopic 3D imaging techniques. ScientificWorld Journal 2009; 9: 1423-37.

[8] Mohun T, Weninger WJ. In: Sharpe J, Wong R, Eds. Imaging in developmental biology: a laboratory manual. NY, USA: Cold Spring Harbor Lab 2010; pp. 765-76.

[9] Weninger WJ, Geyer SH, Mohun TJ, et al. High-resolution episcopic microscopy: a rapid technique for high detailed 3D analysis of gene activity in the context of tissue architecture and morphology. Anat Embryol 2006; 211: 213-21.

[10] Weninger WJ, Mohun T. Phenotyping transgenic embryos: a rapid 3 -D screening method based on episcopic fluorescence image capturing. Nat Genet 2002; 30: 59-65.

[11] Weninger WJ, Geyer SH. Three-dimensional (3D) visualisation of the cardiovascular system of mouse embryos and fetus. Open Cardiovasc Imaging J 2009; 1: 1-12.

[12] Geyer SH, Maurer B, Pötz L, Singh J, Weninger WJ. HighResolution Episcopic Microscopy Data-Based Measurements of the Arteries of Mouse Embryos: Evaluation of Significance and Reproducibility under Routine Conditions. Cells Tissues Organs 2012; 195(6): 524-34.

[13] Weninger WJ, Maurer B, Zendron B, Dorfmeister K, Geyer SH. Measurements of the diameters of the great arteries and semi-lunar valves of chick and mouse embryos. J Microsc 2009; 234: 173-90.

[14] Theiler K. The house mouse: atlas of embryonic development. Springer-Verlag: New York 1989.

[15] Weninger WJ, Mohun TJ. Three-dimensional analysis of molecular signals with episcopic imaging techniques. Methods Mol Biol 2007; 411: 35-46.

[16] Sharpe J. Optical projection tomography as a new tool for studying embryo anatomy. J Anat 2003; 202: 175-81. 
[17] Sharpe J, Ahlgren U, Perry P, et al. Optical projection tomography as a tool for 3D microscopy and gene expression studies. Science 2002; 296: 541-5

[18] Cleary JO, Price AN, Thomas DL, et al. Cardiac phenotyping in ex vivo murine embryos using microMRI. NMR Biomed 2009; 22: $857-66$.

[19] Schneider JE, Bose J, Bamforth SD, et al. Identification of cardiac malformations in mice lacking Ptdsr using a novel high-throughput magnetic resonance imaging technique. BMC Dev Biol 2004; 4: 16.

[20] Metscher BD. MicroCT for comparative morphology: simple staining methods allow high-contrast 3D imaging of diverse nonmineralized animal tissues. BMC Physiol 2009; 9: 11.

[21] Johnson JT, Hansen MS, Wu I, et al. Virtual histology of transgenic mouse embryos for high-throughput phenotyping. PLoS Genet 2006; 2: e61.

(C) Geyer et al:; Licensee Bentham Open.

This is an open access article licensed under the terms of the Creative Commons Attribution Non-Commercial License (http://creativecommons.org/licenses/by-nc/ $3.0 /$ ) which permits unrestricted, non-commercial use, distribution and reproduction in any medium, provided the work is properly cited. 